\title{
The Value of Urine NAG, NGAL Combined with Serum Cys-C in Early Diagnosis of Neonatal Hyperbilirubinemia-related Acute Kidney Injury
}

\author{
Zhen Wang ${ }^{1}$, Lv Jin ${ }^{1}$, Tao Shen ${ }^{1}$, Shihong Zhan ${ }^{2, *}$
}

${ }^{1}$ Department of Neonatology, Suzhou integrated hospital of traditional Chinese and Western Medicine, P. R. China

${ }^{2}$ Department of Neonatology, Children's Hospital of Soochow University,

P. R. China

\section{*Correspondence}

tknf710@163.com

(Shihong Zhan)

\begin{abstract}
Objective: To explore the early diagnostic value of urinary NAG, NGAL and serum Cys-C detection for neonatal hyperbilirubinemia-related acute kidney injury (AKI) in full-term neonates with hyperbilirubinemia. Methodology: One hundred and ninety-six full-term jaundiced newborns were categorized as mild $(n=65)$, moderate $(n=69)$ or severe hyperbilirubinemia $(\mathrm{n}=62)$. The severe group was divided into a non-AKI group $(\mathrm{n}=35)$ and an AKI group $(\mathrm{n}=27)$. Sixty-five full-term newborns with normal serum bilirubin and renal function were analyzed as a normal control group. Urine NAG, urine NGAL and serum Cys-C weremeasured. The value of urinary NAG, NGAL combined with serum Cys-C in early diagnosis of neonatal hyperbilirubinemia-related AKI was evaluated by Receiver Operating Characteristic Curve (ROC). Result: Levels of urine NAG, NGAL and serum Cys-C in the mild, moderateand severe groups were higher than those in the normal control group $(\mathrm{p}<0.001)$, the above indexes levels in the severe group were higher than those in the mildand moderate groups $(\mathrm{p}<0.001)$, and the above indexes levels in the moderate group were higher than those in the mild group $(\mathrm{p}<$ 0.001). Pearson correlation analysis showed that serum Cys-C was positively correlated with urinary NAG and urinary NGAL in AKI group $(r=0.805, p<0.001 ; r=0.864$, $\mathrm{p}<0.001)$; there was a positive correlation between urinary NAG and urinary NGAL in AKI group $(\mathrm{r}=0.948, \mathrm{p}<0.001)$. AUC of urinary NAG, urinary NGAL combined with serum Cys-C in diagnosing neonatal hyperbilirubinemia-related AKI is 0.900 (95\% CI: 0.824-0.976), which is higher than that of urinary NAG, urinary NGAL and serum Cys-C alone. Conclusion: Combined measurement of urine NAG, NGAL and serum Cys-C is helpful for early diagnosis of neonatal hyperbilirubinemia-related AKI.
\end{abstract}

\section{Keywords}

N-Acetyl- $\beta$-D-glucosaminidase (NAG), Neutrophil gelatinase-associated lipid carrier protein (NGAL), Cysteine protease inhibitor C (Cys-C), Hyperbiliubinemia, Acute kidney injury (AKI), Newborn

\section{Introduction}

Neonatal hyperbilirubinemia is one of the common diseases of the newborn period [1]. Previous studies have shown that severe hyperbilirubinemia can induce bilirubin encephalopathy and lead to permanent damage to the nervous system $[2,3]$. One study has found that bilirubin is a potential cause of acute renal tubular necrosis [4]. Some studies have also found that acute kidney injury (AKI) is closely related to hyperbilirubinemia [5]. AKI can induce neonatal death [6]. Clinicians often use oliguria, blood urea nitrogen and creatinine levels as detection methods for kidney injury. Due to the lack of accuracy in the assessment of urine volume in the newborn period, strong renal compensatory ability and low sensitivity in renal function detection, the increase of blood urea nitrogen and creatinine can only be identified when renal function is already seriously damaged, and early detection of renal injury is not easy [7].

Acetyl- $\beta$-D-glucosaminidase (NAG) widely exists in lysosomes of various tissues, organs, body fluids and blood cells, and is one of the most sensitive indicators of renal tubular function [8]. NAG in urine increases significantly when renal tubules are damaged [9]. Some studies have shown that urine NAG may be an appropriate indicator to predict acute renal damage caused by hyperbilirubinemia in full-term newborns [10].

Neutrophil gelatinase-associated lipid carrier protein (NGAL) belongs to the human apolipoprotein superfamily, and directly reflects urinary system epithelial injury. When renal tubular epithelial cells are stimulated by injury, NGAL is significantly induced and highly expressed [11]. The detection 
method of urinary NGAL has the advantages of simplicity, rapidity and non-invasiveness. Some studies have shown that urinary NGAL is helpful for diagnosis of AKI [12].

Cysteine protease inhibitor $\mathrm{C}$ (Cys-C) is an endogenous indicator reflecting glomerular filtration rate [13]. Studies have confirmed that serum Cys-C could be used as a diagnostic tool for AKI $[14,15]$.

The etiology, pathogenesis and pathophysiological process of AKI inpatients with severe hyperbilirubinemia arecomplex, and a single marker cannot comprehensively and accurately reflect the overall changes of AKI. Up to now, there is no specific marker that can be used as an ideal indicator for early diagnosis of neonatal hyperbilirubinemia-related AKI. Therefore, finding a combination of markers that can comprehensively reflect the pathophysiological changes of AKI has become a focus of AKI research related to neonatal hyperbilirubinemia. At present, there is little research on the value of urinary NAG, NGAL combined with serum Cys-C in early the diagnosis of neonatal hyperbilirubinemia-related AKI.

The objective of this study was to evaluate the early diagnostic value of urinary NAG, NGAL and serum Cys-C for neonatal hyperbilirubinemia-related AKI in full-term neonates with hyperbilirubinemia, so as to provide reference values for the early diagnosis and disease assessment of neonatal hyperbilirubinemia-related AKI.

\section{Methodology}

This study was conducted in Suzhou integrated hospital of traditional Chinese and Western Medicine, from September 2017 to March 2020. This study was approved by the ethics committee of Suzhou integrated hospital of traditional Chinese and Western Medicine. One hundred and ninety-six full-term jaundiced newborns were selected as the research subjects. Inclusion criteria were full-term age $\leq 7$ days, no history of asphyxia, infection, dehydration. Exclusion criteria were gestational age $<37$ weeks; hereditary nephropathy, chromosome all disorder maternal systemic lupus erythematosus and other diseases; history of infection, hypoxia and asphyxia; intracranial hemorrhage; newborns receiving nephrotoxic drug therapy and whose mothers have a history of nephropathy. The diagnostic criteria of AKI in KDIGO clinical practice guidelines included rapid decline of renal function within 48 hours and the absolute value of the elevated level of serum creatinine $\geq 26.5 \mu \mathrm{mol} / \mathrm{L}$; or the absolute value of serum creatinine is elevated $\geq 50 \%$ ( 1.5 times $)$ as high as the baseline value; or the urine volume is $<0.5 \mathrm{~mL} \cdot \mathrm{kg}^{-1} \bullet h_{0 u r}{ }^{-1}$ for 6 hours [16].

According to the jaundice intervention standard of Chinese medical association [17], 196 neonates with term jaundice were divided into mild (65 cases) (bilirubin peak value did not meet the phototherapy standard, namely serum total bilirubin $<291 \mu \mathrm{mol} / \mathrm{L}$ ), moderate (69 cases) (bilirubin peak value reached the phototherapy standard but did not meet the exchange blood standard, namely serum total bilirubin $\geq$ $291 \mu \mathrm{mol} / \mathrm{L}$, and serum total bilirubin $<376 \mu \mathrm{mol} / \mathrm{L}$ ) and severe (62 cases) (bilirubin peak value meet the exchange blood standard, namely serum total bilirubin $\geq 376 \mu \mathrm{mol} / \mathrm{L}$, and serum total bilirubin $<428 \mu \mathrm{mol} / \mathrm{L}$ ). The severe group was divided into non-AKI group $(\mathrm{n}=35)$ and AKI group $(\mathrm{n}=$ 27) according to whether the definition of AKI was met. Sixtyfive full-term newborns with normal serum bilirubin and renal function were analyze das normal control group.

All subjects had blood and urine samples drawn within 24 hours after admission. A $4 \mathrm{~mL}$ urine sample was collected by neonatal aseptic urine bag and placed in aseptic test tube for inspection. The 1-2 mL venous blood was collected and sent to dry vacuum tube for inspection. Urine samples and blood samples were tested on the day of sampling.

Urine NAG level was detected by colorimetry, the kit was provided by Beijing Jiuqiang Biotechnology Co., Ltd.. Urine NGAL level was detected by ELISA, the kit was provided by Chongqing Novegent Biotechnology Co., Ltd. Serum Cys-C level was determined by latex immunoturbidimetry, the kit was provided by Beijing Jiuqiang Biotechnology Co., Ltd..

Data were analyzed using SPSS 25.0. KolmogorovSmirnov test or Shapiro-Wilk test was used to test the normality of all measurement data. Measurement data were expressed as mean \pm SD. One-way ANOVA was used to compare the measurement data among groups, and LSD-t test was used to compare the two groups. Measurement data between the two groups were compared by independent sample $t$ test. Pearson correlation analysis was used to analyze the correlation between measurement data conforming to normal distribution. The value of urinary NAG, NGAL combined with serum Cys-C in early diagnosis of neonatal hyperbilirubinemia-related AKI was evaluated by Receiver Operating Characteristic Curve (ROC). A p value less than 0.05 was regarded as statistically significant.

\section{Results}

In the mild group, 31 cases were male (47.69\%) and 34 cases were female $(52.31 \%)$. The mean gestational age was 39.72 \pm 1.35 weeks; the mean age was $5.36 \pm 1.18$ days; The mean birth weight was $3269.25 \pm 381.43 \mathrm{~g}$; Caesarean section was performed in 29 cases (44.62\%) and vaginal delivery in 36 cases (55.38\%); mean serum total bilirubin was $216.72 \pm 5.49$ $\mu \mathrm{mol} / \mathrm{L}$.

In the moderate group, 33 cases were male $(47.83 \%)$ and 36 cases were female $(52.18 \%)$. Mean gestational age was $39.51 \pm 1.62$ weeks; The age was $5.29 \pm 1.23$ days; The mean birth weight was $3301.46 \pm 406.57 \mathrm{~g}$; Caesarean section was performed in 30 cases $(43.48 \%)$ and vaginal delivery in 39 cases $(56.52 \%)$; mean serum total bilirubin was $338.04 \pm 9.21$ $\mu \mathrm{mol} / \mathrm{L}$.

In the severe group, 30 cases were male (48.39\%) and 32 cases were female $(51.61 \%)$. The mean gestational age was $39.16 \pm 1.48$ weeks; The age was $5.22 \pm 1.35$ days; The mean birth weight was $3288.46 \pm 517.29 \mathrm{~g}$; Caesarean section was performed in 27 cases $(43.55 \%)$ and vaginal delivery in 35 cases (56.45\%); mean serum total bilirubin was $397.35 \pm 7.82$ $\mu \mathrm{mol} / \mathrm{L}$.

There were 32 males (49.23\%) and 33 females (50.77\%) in the normal control group. The gestational age was $39.68 \pm$ 1.37 weeks; The age was $5.32 \pm 1.14$ days; The birth weight was $3297.85 \pm 408.54 \mathrm{~g}$; Caesarean section was performed in 30 cases $(46.15 \%)$ and normal delivery in 35 cases $(53.85 \%)$; 
TA B L E 1. Comparison of urinary NAG, NGAL and serum Cys-C in 4 groups.

\begin{tabular}{|lccccc|} 
Parameter & $\begin{array}{c}\text { Normal control } \\
\text { group }(\mathbf{n}=\mathbf{6 5})\end{array}$ & $\begin{array}{c}\text { Mild group } \\
(\mathbf{n = 6 5})\end{array}$ & $\begin{array}{c}\text { Moderate group } \\
(\mathbf{n}=\mathbf{6 9})\end{array}$ & $\begin{array}{c}\text { Severe group } \\
(\mathbf{n}=\mathbf{6 2})\end{array}$ & p value \\
\hline Urine NAG $(\mathrm{U} / \mathrm{L})$ & $8.63 \pm 0.83$ & $10.17 \pm 1.54$ & $12.01 \pm 1.77$ & $14.44 \pm 2.12$ & $<0.001$ \\
\hline Urine NGAL $(\mu \mathrm{g} / \mathrm{L})$ & $2.50 \pm 0.48$ & $4.76 \pm 0.75$ & $7.02 \pm 1.30$ & $8.47 \pm 2.70$ & $<0.001$ \\
\hline Serum Cys-C $(\mathrm{mg} / \mathrm{L})$ & $0.78 \pm 0.09$ & $1.07 \pm 0.14$ & $1.25 \pm 0.18$ & $1.52 \pm 0.24$ & $<0.001$ \\
\hline
\end{tabular}

T A B L E 2. Comparison of urinary NAG, NGAL and serum Cys-C between AKI group and non-AKI group.

\begin{tabular}{lccc} 
Parameter & Non-AKI group $(\mathbf{n}=\mathbf{3 5})$ & AKI group $(\mathbf{n}=\mathbf{2 7})$ & p value \\
\hline Urine NAG $(\mathrm{U} / \mathrm{L})$ & $13.57 \pm 1.74$ & $15.58 \pm 2.06$ & $<0.001$ \\
Urine NGAL $(\mu \mathrm{g} / \mathrm{L})$ & $6.49 \pm 1.13$ & $12.03 \pm 1.28$ & $<0.001$ \\
\hline Serum Cys-C $(\mathrm{mg} / \mathrm{L})$ & $1.40 \pm 0.26$ & $1.70 \pm 0.14$ & $<0.001$ \\
\hline
\end{tabular}

serum total bilirubin was $24.31 \pm 6.02 \mu \mathrm{mol} / \mathrm{L}$.

The levels of urine NAG, NGAL and serum Cys-C in the mild group, moderate group and severe group were higher than those in the normal control group ( $<<0.001)$, the levels of all indices in the severe group were higher than those in mild group and moderate group $(\mathrm{p}<0.001)$, and the levels in the moderate group were higher than those in mild group $(\mathrm{p}<$ 0.001), as shown in Table 1.

Urine NAG, NGAL and serum Cys-C levels in AKI group were higher than those in non-AKI group $(\mathrm{p}<0.001)$, as shown in Table 2.

Pearson correlation analysis showed that serum Cys-C was positively correlated with urinary NAG and urinary NGAL in AKI group $(\mathrm{r}=0.805, \mathrm{p}<0.001$; there was a positive correlation between urinary NAG and urinary NGAL in AKI group $(\mathrm{r}=0.948, \mathrm{p}<0.001)$.

The AUC of urinary NAG, urinary NGAL and serum Cys$\mathrm{C}$ in diagnosing neonatal hyperbilirubinemia-related AKI were 0.754 (95\% CI: $0.634-0.875), 0.748$ (95\% CI: $0.623-0.873)$ and 0.844 (95\% CI: $0.751-0.938)$, respectively. When the critical value of urinary NAG was $15.26 \mathrm{U} / \mathrm{L}$, the sensitivity and specificity were $55.60 \%$ and $85.70 \%$, respectively. When the critical value of urinary NGAL was $8.14 \mathrm{~g} / \mathrm{L}$, its diagnostic sensitivity was $74.10 \%$ and specificity $71.40 \%$. When the critical value of serum Cys-C was $1.55 \mathrm{mg} / \mathrm{L}$, its diagnostic sensitivity was $85.20 \%$ and specificity $62.90 \%$. The AUC of urinary NAG, urinary NGAL combined with serum Cys-C in diagnosing neonatal hyperbilirubinemia-related AKI is 0.900 (95\% CI: $0.824-0.976)$, which is higher than that of urinary NAG, urinary NGAL and serum Cys-C alone. Its diagnostic sensitivity is $77.80 \%$, and its specificity is $88.60 \%$, as shown in Fig. 1.

\section{Discussion}

Hyperbilirubinemia may cause damage to neonatal kidneys due to the increase of serum free bilirubin [18]. Free bilirubin is fat soluble and can penetrate through cell membrane and enter renal cells, which can interfere with renal cell metabolism and cause renal function changes [19].

Some studies have shownthat serum SCr and BUN have poor sensitivity and low diagnostic efficiency in predicting neonatal hyperbilirubinemia-related AKI [20]. A study has
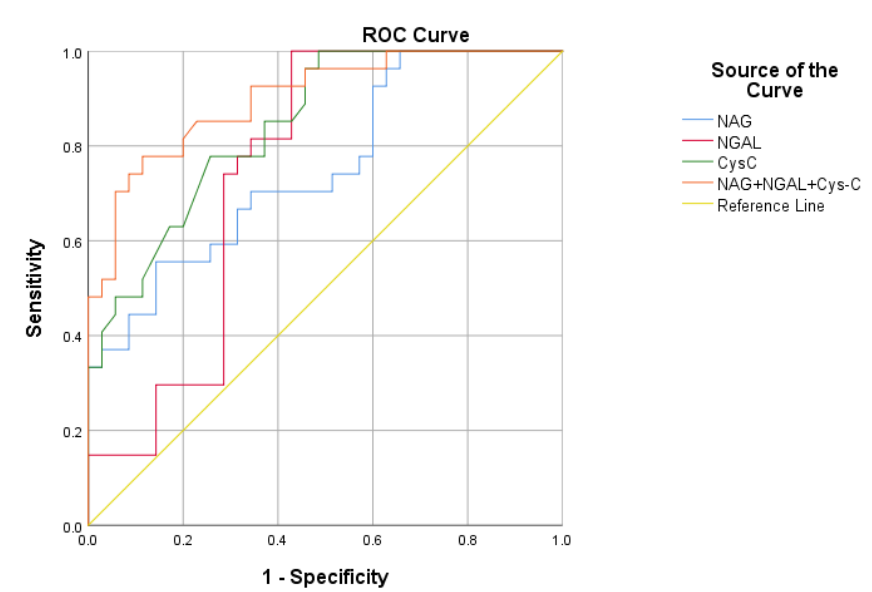

F I G U RE 1. ROC curve analysis results.

shown that serum Cys-C is more sensitive to renal function damage than $\mathrm{SCr}$, and is an ideal homologous marker reflecting changes in glomerular filtration rate [21].

NAG widely exists in lysosomes of various tissues, organs, body fluids and blood cells, and is one of the most sensitive indicators of renal tubular function. NAG in urine increased significantly when renal tubules were damaged [22]. NAG is a sensitive indicator of renal tubular injury [23]. Katagiri et al. confirmed combined detection of urinary L-type fatty acid-binding protein and NAG could detect adult post-cardiac surgery AKI [24]. The results of this study showed that the urine NAG levels in the mild, moderate and severe groups were higher than those in normal control group, the urine NAG levels in severe group were higher than those in mild group, and the urine NAG levels in moderate group were higher than those in mild group. Urine NAG level in AKI group was higher than that in non-AKI group. It is suggested that urinary NAG can be used to diagnose AKI of full-term newborns.

Some studies have shown that the specificity of urinary NAG in diagnosing diabetic renal damage is not as good as that of urinary NGAL [25]. The biological function of NGAL has not been clearly defined. NGAL is an acute phase reactive protein, which may participate in chemotaxis, immune response and reduce tissue damage caused by oxidative stress. Studies have found that renal proximal convoluted tubule cells can highly express NGAL after injury [26]. Some studies have 
also found that exogenous NGAL protein plays a protective role in ischemia/reperfusion injury, providing a new way for the prevention and treatment of renal ischemia/reperfusion injury [27]. Kari et al. demonstrated that urinary NGAL and serum Cys-C could predict AKI early in children admitted to an intensive care unit [28]. The results of our study showed that the urine NGAL level of the mild group, moderate group and severe group was higher than that of normal control group, the urine NGAL level of severe group was higher than that of mild group, and the urine NGAL level of moderate group was higher than that of mild group. Urine NGAL level in AKI group was higher than that in non-AKI group. It is suggested that urinary NGAL can be used to diagnose AKI in full-term newborns. Some studies have also found that urinary NGAL is significantly related to birth weight and gestational age of premature infants [29]. Urine NGAL as a biomarker of AKI in premature infants deserves further study.

Correlation analysis showed that urinary NAG, NGAL and serum Cys-C were positively correlated, which further shows that urinary NAG, NGAL and serum Cys-C were sensitive indicators reflecting early renal function damage.

Combined marker monitoring is helpful to improve the efficiency of predicting AKI in severe patients. However, it is still unclear which markers are the most ideal combination for joint detection. Using ROC curve analysis, the AUC of urinary NAG, NGAL combined with serum Cys-C in predicting neonatal hyperbilirubinemia-related AKI was higher than that of urinary NAG, urinary NGAL and serum Cys-C alone, and the sensitivity and specificity were higher, which was a reliable index for predicting neonatal hyperbilirubinemia complicated with AKI. However, the sample size of this study was small, and a large sample and multi-center clinical study are needed to further verify the clinical value of the combined detection of the above three markers. Different from adults and children, newborns' kidney is not yet mature and their renal function is influenced by a number of factors, such as gestational age and birth weight. Therefore, the results of this study may be affected by these confounding factors, such as gestational age and birth weight. In future, the influence brought by the above confounding factors should be examined via multiple logistic regression analysis, and whether urinary NAG, NGAL and serum Cys-C are an independent predictive index of AKI associated with neonatal hyperbilirubinemia shall be determined.

\section{Conclusion}

Combined detection of urine NAG, NGAL and serum Cys-C is helpful for early diagnosis of neonatal hyperbilirubinemiarelated AKI.

\section{ACKNOWLEDGEMENTS}

I would like to express my gratitude to all those who helped me during the writing of this manuscript. Thanks to all the peer reviewers and editors for their opinions and suggestions.

\section{CONFLICT OF INTEREST}

The authors have no conflicts of interests to declare.

\section{REFERENCES}

[1] Shan KH, Wang TM, Lin MC. Association between rooming-in policy and neonatal hyperbilirubinemia. Pediatr Neonatol. 2019;60:186-191.

[2] Bhardwaj K, Locke T, Biringer A, et al. Newborn Bilirubin Screening for Preventing Severe Hyperbilirubinemia and Bilirubin Encephalopathy: A Rapid Review. Curr Pediatr Rev. 2017;13:67-90.

[3] Bech LF, Donneborg ML, Lund AM, et al. Extreme neonatal hyperbilirubinemia, acute bilirubin encephalopathy, and kernicterus spectrum disorder in children with galactosemia. Pediatr Res. 2018;84:228-232.

[4] Rafat C, Burbach M, Brochériou I, et al. Bilirubin-Associated Acute Tubular Necrosis in a Kidney Transplant Recipient. Am J Kidney Dis. 2013;61:782-785.

[5] Wu YH, Wu CY, Cheng CY, et al. Severe Hyperbilirubinemia Is Associated With Higher Risk of Contrast-Related Acute Kidney Injury Following Contrast-Enhanced Computed Tomography. PLoS One. 2020;15:e0231264.

[6] Durkan AM, Alexander RT. Acute Kidney Injury Post Neonatal Asphyxia. J Pediatr. 2011;158:e29-33.

[7] Srichai MB, Hao C, Davis L, et al. Apoptosis of the Thick Ascending Limb Results in Acute Kidney Injury. J AM Soc Nephrol. 2008;19:15381546.

[8] Skálová S. The Diagnostic Role of Urinary N-acetyl-beta-Dglucosaminidase (NAG) Activity in the Detection of Renal Tubular Impairment. Acta Medica (Hradec Kralove). 2005;48:75-80.

[9] Kim YD, Yim DH, Eom SY, et al. Temporal Changes in Urinary Levels of Cadmium, $\mathrm{N}$-acetyl- $\beta$-d-glucosaminidase and $\beta 2$-microglobulin in Individuals in a Cadmium-Contaminated Area. Environ Toxicol Pharmacol. 2015;39:35-41.

[10] Cheng B, Jin Y, Liu G, et al. Urinary N-acetyl-beta-D-glucosaminidase as an early marker for acute kidney injury in full-term newborns with neonatal hyperbilirubinemia. Dis Markers. 2014;2014:315843.

[11] Gong L, Yu H, Zhu Ge Y, et al. Neutrophil Gelatinase-Associated Lipocalin Protects Renal Tubular Epithelial Cell in ischemic/reperfusion Injury Rats via Apoptosis-Regulating Proteins. Ren Fail. 2012;34:777783.

[12] Segawa H, Hatta T, Kawasaki Y, et al. The utility of urinary NGAL (neutrophil gelatinase-associated lipocalin) as a prognostic marker for initiating acute hemoperfusion therapy. Nihon Toseki Igakkai Zasshi. 2014;47:487-491.

[13] Sjöström P, Tidman M, Jones I. Determination of the production rate and non-renal clearance of cystatin $\mathrm{C}$ and estimation of the glomerular filtration rate from the serum concentration of cystatin $\mathrm{C}$ in humans. Scand J Clin Lab Invest. 2005;65:111-124.

[14] Bagshaw SM, Bellomo R. Cystatin C in Acute Kidney Injury. Curr Opin Crit Care. 2010;16:533-539.

[15] Kari JA, Shalaby MA, Sofyani K, et al. Urinary neutrophil gelatinaseassociated lipocalin (NGAL) and serum cystatin C measurements for early diagnosis of acute kidney injury in children admitted to PICU. World J Pediatr. 2018;14:134-142.

[16] Khwaja A. KDIGO clinical practice guidelines for acutekidney injury. Nephron Clin Pract. 2012;120:c179-184.

[17] Ding G, Pu M. Recommendations intervention for neonatal jaundice. Chin J paediatr. 2001;39:185-187.

[18] Lund HT, Jacobsen J. Influence of Phototherapy on Unconjugated Bilirubin in Duodenal Bile of Newborn Infants With Hyperbilirubinemia. Acta Paediatr Scand. 1972;61:693-696.

[19] Ahlfors CE, Wennberg RP, Ostrow JD, et al. Unbound (free) bilirubin: improving the paradigm for evaluating neonatal jaundice. Clin Chem. 2009;55:1288-1299.

[20] Edelstein CL. Biomarkers in Acute Kidney Injury. Biomarkers of Kidney Disease (Second edition). 2017. p. 241-315.

[21] Kiyosue A, Hirata Y, Ando J, et al. Plasma Cystatin C Concentration Reflects the Severity of Coronary Artery Disease in Patients Without Chronic Kidney Disease. Circ J. 2010;74:2441-2447.

[22] Bosomworth MP, Aparicio SR, Hay AW. Urine N-acetyl-beta-Dglucosaminidase-a marker of tubular damage? Nephrology Dialysis Transplantation. 1999;14:620-626. 
[23] Salem MA, el-Habashy SA, Saeid OM, et al. Urinary excretion of nacetyl-beta-D-glucosaminidase and retinol binding protein as alternative indicators of nephropathy in patients with type 1 diabetes mellitus. Pediatric Diabetes. 2002;3:37-41.

[24] Katagiri D, Doi K, Honda K, et al. Combination of two urinary biomarkers predicts acute kidney injury after adult cardiac surgery. Ann Thorac Surg. 2012;93:577-583.

[25] Fu W, Xiong S, Fang Y, et al. Urinary tubular biomarkers in short-term type 2 diabetes mellitus patients: a cross-sectional study. Endocrine. 2012;41:82-88

[26] Zhou X, Ma B, Lin Z, et al. Evaluation of the Usefulness of Novel Biomarkers for Drug-Induced Acute Kidney Injury in Beagle Dogs. Toxicol Appl Pharmacol. 2014;280:30-35.

[27] Zhang Y, Qiao S, Wang R, et al. NGAL Attenuates Renal ischemia/reperfusion Injury Through Autophagy Activation and Apoptosis
Inhibition in Rats. Chem Biol Interact. 2018;289:40-46.

[28] Kari JA, Shalaby MA, Sofyani K, et al. Urinary neutrophil gelatinaseassociated lipocalin (NGAL) and serum cystatin $\mathrm{C}$ measurements for early diagnosis of acute kidney injury in children admitted to PICU. World J Pediatr. 2018;14:134-142.

[29] Lavery AP, Meinzen-Derr J K, Anderson E, et al. Urinary NGAL in Premature Infants. Pediatr Res. 2008;64: 423-428.

How to cite this article: Zhen Wang, Lv Jin, Tao Shen, Shihong Zhan. The Value of Urine NAG, NGAL Combined with Serum Cys-C in Early Diagnosis of Neonatal Hyperbilirubinemiarelated Acute Kidney Injury. Signa Vitae. 2020;16(2):109-113. doi:10.22514/sv.2020.16.0055. 